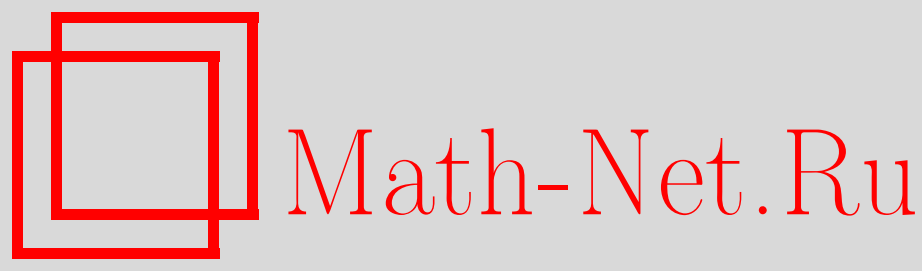

Д. Р. Ведагедера, О фазовой диаграмме направленного полимера в случайной среде с $p$-спиновым ферромагнитным взаимодействием, ТМФ, 2011, том 166, номер 1, 121-141

DOI: https://doi.org/10.4213/tmf6600

Использование Общероссийского математического портала Math-Net.Ru подразумевает, что вы прочитали и согласны с пользовательским соглашением http://www.mathnet.ru/rus/agreement

Параметры загрузки:

IP : 52.87 .193 .239

26 апреля 2023 г., 12:42:56

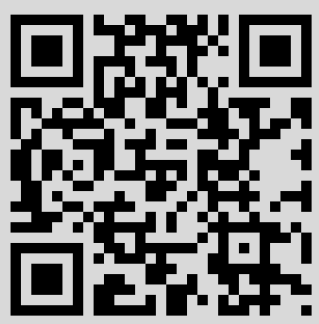




\title{
О ФАЗОВОЙ ДИАГРАММЕ НАПРАВЛЕННОГО ПОЛИМЕРА В СЛУЧАЙНОЙ СРЕДЕ С $p$-СПИНОВЫМ ФЕРРОМАГНИТНЫМ ВЗАИМОДЕЙСТВИЕМ
}

\begin{abstract}
Рассматривается модель направленного полимера с аддитивным $p$-спиновым ферромагнитным членом $(p>2)$ в гамильтониане. Приведено строгое доказательство для специфической свободной энергии, и получена ее фазовая диаграмма. Эта модель была предложена ранее и было дано подробное доказательство для случая $p=2$, в то время как для случая $p>2$ был сформулирован только основной результат. Здесь дано подробное доказательство основного результата и продемонстрировано поведение модели при $p \rightarrow \infty$ путем построения фазовой диаграммы также и в этом случае. Эти результаты являются важными для многих приложений, например для телекоммуникаций и иммунологии. Основной результат состоит в том, что на фазовой диаграмме для $p>2$ возникает новая кривая переходов, отсутствующая для $p=2$, между парамагнитной областью и так называемой смешанной областью, а также в том, что ферромагнитная область уменьшается при $p \rightarrow \infty$.
\end{abstract}

Ключевые слова: направленный полимер, большие уклонения, фазовая диаграмма.

\section{1. ВВЕДЕНИЕ}

Направленные полимеры в случайных средах (самоизбегающие случайные блуждания в случайных средах или случайных деревьях [1]) широко применяются не только в физических науках, но также и во многих других областях (например, динамика общественного мнения и финансовый рынок [2]-[5], эволюционные динамики [6], [7], агрегация, ограниченная диффузией [8], возмущения в одномерной турбулентности [9], [10], иммунология [11]-[13] и т.д.).

Направленный полимер на разупорядоченном дереве является примером диффузии в разупорядочных средах [14]-[16] и моделью случайной энергии (МСЭ) Дерриды [17]-[21], которая состоит из $z^{n}$ независимо и тождественно распределенных уровней случайной энергии дерева, которое делится на $z$ ветвей в каждом узле этого дерева (см. рис. 1 для случая $z=2$ ). Действительно, в работах [22], [19] показано, что МСЭ является предельным случаем модели Шеррингтона-Киркпатрика

* Department of Mathematics, University of Ruhuna, Matara, Sri Lanka.

E-mail: janak@maths.ruh.ac.lk 
с взаимодействием $r$ спинов при $r \rightarrow \infty$. Взаимодействие $r$ спинов определяется гамильтонианом

$$
-\mathcal{H}_{\mathrm{MC} \ni}=\sum_{i_{1}<i_{2}<\cdots<i_{r}=1}^{n} J_{i_{1}, i_{2}, \ldots, i_{r}} \sigma_{i_{1}} \sigma_{i_{2}} \ldots \sigma_{i_{r}}
$$

где $J_{i_{1}, i_{2}, \ldots, i_{r}}$ - независимые и тождественно распределенные гауссовские случайные переменные (замороженные, что характеризует обменные интегралы), $\sigma_{i_{1}}, \ldots, \sigma_{i_{r}}-$ спины Изинга. Если $r$ мало $(r \ll n)$, то можно ожидать, что уровни энергии, возникшие при взаимодействии $r$ спинов, будут сильно скоррелированными, в то время как эти корреляции станут пренебрежимо малыми при $r \rightarrow \infty$; в таком случае восстанавливается МСЭ - результирующие $2^{n}$ независимые и тождественно распределенные уровни энергии, для которых

$$
-\mathcal{H}_{\mathrm{MC} \ni}=\sum_{i=1}^{n} V_{i}
$$

Основным объектом, интересующим нас в этой статье, является направленный полимер, который определяется как самоизбегающее случайное блуждание вдоль дерева, которое разветвляется на $z=2$ направлений случайным образом в каждом узле и имеет базовый гамильтониан, описываемый так же, как и гамильтониан MCЭ (1).

Рассмотрим пример, изображенный на рис. 1 , где мы находим $2^{n}$ независимых и тождественно распределенных энергетических уровня, каждый из которых соответствует одному из $2^{n}$ блужданий (или путей по нашему определению; см. ниже), заканчивающемуся после $n$ шагов, вдоль дерева, показанного на рис. 1б. Такое блуждание может быть получено, например, при рассмотрении системы, состоящей из $n$ локализованных спинов Изинга $\sigma_{1}, \sigma_{2}, \ldots, \sigma_{n}$. Можно определить энергию каждого блуждания как сумму энергий, соответствующих каждой ветви, которую пересекает это блуждание. Мы можем определить гамильтониан для этого случая в виде

$$
-\mathcal{H}_{1}:=\sum_{j=1}^{n} V_{j,(\sigma)^{j}}
$$

для данной конфигурации спинов, например $(\sigma)^{j}$, и для величин $V$, имеющих некоторое предыдущее распределение вероятности, зависящее от одного параметра, скажем $\gamma$. Кроме того, каждая $p$-группа этих спинов могла бы оказывать в системе ферромагнитное воздействие (вследствие воздействия какого-то внешнего поля или из-за взаимодействия $p$-групп; см. рис. 1), которое мы описываем с помощью гамильтониана

$$
-\mathcal{H}_{2}:=\frac{1}{n^{p-1}} \sum_{k=1}^{M} h_{k}\left|\sum_{j=1}^{n} \sigma_{j}^{(k)}\right|^{p}, \quad p>1,
$$

который можно упростить следующим образом:

$$
-\mathcal{H}_{2}=\frac{1}{n^{p-1}}\left(h_{1}+h_{2}+\cdots+h_{M}\right)\left|\sum_{j=1}^{n} \sigma_{j}\right|^{p}=\frac{1}{n^{p-1}} \lambda\left|\sum_{j=1}^{n} \sigma_{j}\right|^{p},
$$




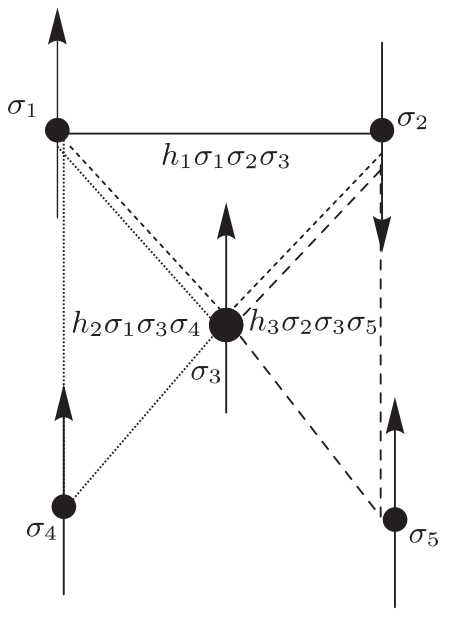

a

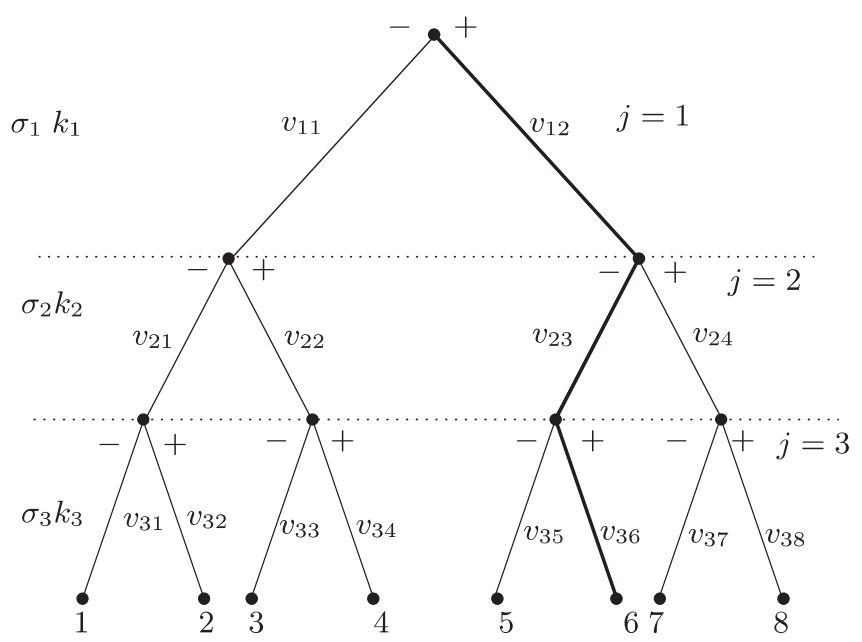

6

Рис. 1. Образцовый случай возникновения $2^{n}$ возможных энергетических уровней: спины Изинга $\sigma_{1}, \ldots, \sigma_{n}$ (здесь $n=5$ ) оказывают ферромагнитное воздействие на систему со стороны $p$-спиновой группы $(p=3)$; это определяется с помощью $\sum_{l=1}^{\left(\begin{array}{l}5 \\ 3\end{array}\right)} h_{l} \sum_{i<j<k ; i, j, k=1}^{5} \sigma_{i} \sigma_{j} \sigma_{k}+\left(\sum_{i=1}^{5} \sigma_{i}\right)^{3}-$ (члены низкого порядка) (а). Структура энергетических уровней в виде дерева: $k_{j+1}=2 k_{j}-\left(1-\sigma_{j+1}\right) / 2, k_{j}=1,2, \ldots, n$ и $\sigma_{i}= \pm 1$ в случае $n=3$; суммарная энергия системы состоит из энергии каждой конфигурации спинов и энергии взаимодействия $p$-групп, показаны только три первых уровня этого пути (б).

так как конфигурации $\sigma_{j}^{(k)}$ являются неразличимыми. Здесь величина $\lambda:=\sum_{k=1}^{M} h_{k}$ может быть либо параметром, либо замороженной случайной переменной, а $M=\left(\begin{array}{c}N \\ p\end{array}\right)$. Полный гамильтониан для этой системы записывается в виде $\mathcal{H}=\mathcal{H}_{1}+\mathcal{H}_{2}$. Действительно, основываясь на приведенном выше примере, мы определим полную модель в разделе 2 .

Модель, которую мы рассматриваем здесь, решалась для специфической свободной энергии в работе [23], где приведено доказательство только для случая $p=2$ с использованием аргумента, связанного с большим уклонением уровня I для мартингал-процесса (как и в работе [24]), определенного в виде

$$
M_{n}(\beta, t)=\sum_{\left\{\sigma_{j}\right\}_{j=1}^{n}} \exp \left\{\beta \sum_{j=1}^{n} V_{j,(\sigma)^{j}}+t \sum_{j=1}^{n} \sigma_{j}\right\} / \operatorname{ch}(t) \mathbb{E}\left\{e^{\beta V}\right\}
$$

где $\beta$ и $t$ являются параметрами.

Действительно, в работе [23] утверждалось, что фазовая диаграмма для случая $p>2$ может быть определена так же, как и в случае $p=2$, а подробности можно найти в работе [25]. Однако эти подробности не опубликованы до сих пор, и, так как 
модель для случая $p>2$ (как отмечено выше и в разделе 6) может использоваться в других контекстах, результаты мы постараемся представить максимально подробно.

Статья организована следующим образом.

В разделе 2 мы еще раз сформулируем определение модели, терминологию и главные результаты, доказанные в работе [23]. В разделе 3 мы выводим функции распределения (вариационная задача). В разделе 4 мы приводим основной результат этой статьи - теорему 2, перед которой даем ряд лемм и предложений, необходимых для того, чтобы обосновать фазовую диаграмму (см. ниже рис. 2 и рис. 6). И, наконец, в разделе 5 мы получаем фазовую диаграмму модели в случае $p \rightarrow \infty$.

\section{2. ТЕРМИНОЛОГИЯ И ОПРЕДЕЛЕНИЯ}

Для полноты изложения мы повторим некоторые определения и результаты работы [23], в которой отсутствуют подробности доказательства основной теоремы (для случая $p>2$ ).

Рассмотрим дерево Кэли (рис. 1) с координационным числом 3, т. е. каждый узел этого дерева соединен с другими двумя на следующем уровне. Обозначим связи дерева с помощью $(j, k)$, где $j, k \in \mathbb{N}$, и $j$ соответствует поколению, а $k \in\left\{1, \ldots, 2^{j}\right\}-$ связям слева направо внутри $j$-го поколения. Каждой связи дерева мы припишем независимые, тождественно распределенные переменные $V_{j, k}$, которые имеют известное распределение.

Путь длиной $n$, начинающийся на вершине дерева, определяется как конечная последовательность

$$
\left\{\left(j, k_{j}\right) ; 1 \leqslant j \leqslant n\right\}
$$

удовлетворяющая соотношению

$$
k_{j+1}=2 k_{j}-\frac{1}{2}\left(1-\sigma_{j+1}\right),
$$

где $\sigma_{j} \in\{-1,1\}$ соответствуют изъятию левой или правой ветви из $j$-го поколения (см. рис. 1). Мы обозначим последовательность спинов Изинга $\left(\sigma_{k}\right)_{k=1}^{j}$ через $(\sigma)^{j}$. Тогда путь будет полностью определен с помощью $(\sigma)^{n}$.

Наша главная задача заключается в получении замкнутого выражения для специфической свободной энергии и фазовой диаграммы для модели.

Мы будем определять наблюдаемые (или параметры порядка) для этой модели на пространстве конфигураций $X^{n}:=\{\sigma: \Lambda \rightarrow X\}$, где $X$ - компактное метрическое пространство, а $\Lambda=\{1, \ldots, n\}$, где $n$ - любое неотрицательное целое число. В этом частном случае мы положим $X=\{-1,1\}$. Определим распределение одного спина $\mu(\{\sigma\})=1 / 2, \sigma= \pm 1$, а меру $n$-кратного произведения $\mu$ обозначим как $\mathbb{F}_{n}^{\mu}$. Тогда наблюдаемые $V_{n}: X^{n} \rightarrow \mathbb{R}, m_{n}: X^{n} \rightarrow \mathbb{R}$ имеют вид

$$
V_{n}(\sigma)=\frac{1}{n} \sum_{j=1}^{n} V_{j,(\sigma)^{j}}, \quad m_{n}(\sigma)=\frac{1}{n} \sum_{j=1}^{n} \sigma_{j} .
$$

Гамильтониан определяется в виде

$$
-\mathcal{H}=\sum_{j=1}^{n} V_{j,(\sigma)^{j}}+\frac{\lambda}{n^{p-1}}\left|\sum_{j=1}^{n} \sigma_{j}\right|^{p},
$$

где $p \geqslant 2-$ произвольный параметр, $\lambda>0-$ константа связи. 
Таким образом, функиия разбиения $\mathcal{Z}_{n}$ имеет следующее разложение:

$$
\mathcal{Z}_{n}=\sum_{\left\{\sigma_{j}\right\}_{j=1}^{n}} e^{-\beta \mathcal{H}}=\sum_{\left\{\sigma_{j}\right\}_{j=1}^{n}} \exp \left\{\beta n\left[\frac{1}{n} \sum_{j=1}^{n} V_{j,(\sigma)^{j}}+\lambda\left(\frac{1}{n} \sum_{j=1}^{n} \sigma_{j}\right)^{p}\right]\right\} .
$$

Специфическая свободная энергия системы определяется выражением

$$
-\beta f(\beta, \lambda, \gamma):=\lim _{n \rightarrow \infty} \frac{1}{n} \ln \mathcal{Z}_{n}(\beta) .
$$

Заметим, что поскольку $V_{j(\sigma)^{j}}$ зависит от всех предыдущих $\sigma_{k}, k \leqslant j$, нам необходимо использовать мартингальные свойства [26], связанные с $\mathcal{Z}_{n}$, чтобы вычислить суму по $\left\{\sigma_{j}\right\}$ в $(8)$.

Теперь мы можем записать свободную энергию (9) в виде

$$
-\beta f(\beta, \lambda, \gamma)=\lim _{n \rightarrow \infty} \frac{1}{n} \ln \mathcal{Z}_{n}(\beta)=\lim _{n \rightarrow \infty} \frac{1}{n} \ln \int_{X_{n}} e^{-\beta \mathcal{H}} \mathbb{F}_{n}^{\mu}(d \sigma)+\ln 2 .
$$

Преобразуем интеграл $(10)$ в интеграл по мере образа $\mathbb{Q}_{n}$ меры $\mathbb{F}_{n}$, индуцированной отображением $W_{n}=\left(V_{n}, m_{n}\right)$ и определенной в виде

$$
\mathbb{Q}_{n}(A)=\mathbb{F}_{n}^{\mu}\left(W_{n}^{-1}(A)\right) \quad \forall A \in \mathcal{B}\left(\mathbb{R}^{2}\right)
$$

следующим образом:

$$
-\beta f(\beta, \lambda, \gamma)=\lim _{n \rightarrow \infty} \frac{1}{n} \ln \int_{\mathbb{R}^{2}} e^{n \beta\left(v+\lambda m^{p}\right)} \mathbb{Q}_{n}(d v, d m)+\ln 2,
$$

где $(v, m) \in A \subset \mathcal{B}\left(\mathbb{R}^{2}\right)$. Предел в (12) можно вычислить с помощью теоремы Варадхана [27]:

$$
-\beta f(\beta, \lambda, \gamma)=\sup _{v, m}\left\{\beta\left(v+\lambda m^{p}\right)-I(v, m)\right\}+\ln 2,
$$

где

$$
I(v, m)=\sup _{t_{1}, t_{2}}\left\{t_{1} v+t_{2} m-C\left(t_{1}, t_{2}\right)\right\}
$$

называется функцией распределения, а $C\left(t_{1}, t_{2}\right)$ - кумулянтная производящая функиия, определяемая в виде

$$
C\left(t_{1}, t_{2}\right)=\lim _{n \rightarrow \infty} \frac{1}{n} \ln \int e^{t_{1} v+t_{2} m} \mathbb{Q}_{n}(d v, d m) .
$$

Таким образом, основная задача заключается в вычислении кумулянтной производящей функции.

Действительно, из приведенного выше изложения результатов работы [23] нетрудно видеть, что $t_{1} \equiv \beta$. Положив

$$
I^{\beta}(m):=\sup _{t}\{t m-C(\beta, t)\},
$$

можно переписать выражение для свободной энергии (13) в виде

$$
-\beta f(\beta, \lambda)=\ln 2+\sup _{m}\left\{\lambda \beta m^{p}-I^{\beta}(m)\right\},
$$

при этом используется выпуклость кумулянтной производящей функции. 
В работе [23] была доказана следующая теорема (гарантирующая существование предела в (14)), которая основывается на том факте, что

$$
M_{n}(\beta, m)=\sum_{\left\{\sigma_{j}\right\}_{j=1}^{n}} \exp \left\{\beta \sum_{j=1}^{n} V_{j,(\sigma)^{j}}+m \sum_{j=1}^{n} \sigma_{j}\right\} / \operatorname{ch}(m) \mathbb{E}\left\{e^{\beta V}\right\}
$$

является мартингалом относительно возрастающего семейства случайных переменных $\nu^{n}=\left\{V_{j,(\sigma)^{j}} ; 1 \leqslant k \leqslant 2^{j}, 1 \leqslant j\right\}$.

Определим функции

$$
\begin{gathered}
\bar{\beta}(t):=\{2 \gamma[\ln (2 \operatorname{ch} t)-t \operatorname{th} t]\}^{1 / 2}, \quad T(t):=\bar{\beta}^{-1}, \quad \beta_{0}:=\bar{\beta}(0)=\sqrt{2 \gamma \ln 2} \\
\Gamma(t):=\left\{(\beta, t) \in \mathbb{R}^{2}:|t|<T(\beta)\right\} .
\end{gathered}
$$

Теорема 1 [23]. Предположим, что $V$ подчиняется распределению Гаусса с нулевым средним и дисперсией $1 / \gamma$. Тогда

$$
C(\beta, t)= \begin{cases}C_{1}(\beta, t):=\ln (2 \operatorname{ch} t)+\beta^{2} /(2 \gamma)-\ln 2, & \beta \leqslant \bar{\beta}(t), \\ C_{2}(\beta, t):=(\beta / \bar{\beta})\left[\ln (2 \operatorname{ch} \bar{t})+\bar{\beta}^{2} /(2 \gamma)\right]-\ln 2, & \beta \geqslant \bar{\beta}(t)\end{cases}
$$

где $\bar{t}$ является решением уравнения

$$
\bar{t}=\frac{\bar{\beta} t}{\beta} .
$$

\section{3. ФУНКЦИЯ РАСПРЕДЕЛЕНИЯ}

В работе [23] было дано краткое изложение доказательства следующего результата. Приведем его здесь подробно.

ПРЕДЛОЖЕНИЕ 1. Пусть $m(\beta):=\operatorname{th}[T(\beta)]$,

$$
I_{1}^{\beta}(m)=\sup _{0 \leqslant t \leqslant T(\beta)}\left\{t m-C_{1}(\beta, t)\right\}
$$

$u$

$$
I_{2}^{\beta}(m)=\sup _{T(\beta) \leqslant t}\left\{t m-C_{2}(\beta, t)\right\}
$$

Тогда

$$
I^{\beta}(m)= \begin{cases}I_{1}^{\beta}(m)=I_{0}(m)-\beta^{2} /(2 \gamma), & 0 \leqslant|m| \leqslant m(\beta), \\ I_{2}^{\beta}(m)=-\beta \bar{\beta} / \gamma+\ln 2, & m(\beta)<|m|<1, \\ \infty & \text { в остальных случаях. }\end{cases}
$$

ДокАЗАтельство. Заметим, что поскольку $I^{\beta}(m)=\max \left\{I_{1}^{\beta}, I_{2}^{\beta}\right\}$, то нужно определить, какая из величин $I_{1}^{\beta}$ и $I_{2}^{\beta}$ доминирует и когда это произойдет. Сначала предположим, что $t \leqslant T(\beta)$ и $\beta<\beta_{0}$. Тогда с помощью условия максимизации для (20) мы получаем

$$
m=\frac{\partial}{\partial t} C_{1}(\beta, t)=\operatorname{th} t
$$

Отсюда следует, что $m \leqslant m(\beta)$. 
Если $\tilde{t}$ - максимайзер функции (20), то мы имеем $\partial I_{1} / \partial m=\tilde{t}$, и, следовательно,

$$
\begin{aligned}
I_{1}^{\beta}(m) & =m \operatorname{th}^{-1} m-C_{1}\left(\beta, \operatorname{th}^{-1} m\right)= \\
& =m \operatorname{th}^{-1} m-\ln \left[2 \operatorname{ch}\left(\operatorname{th}^{-1} m\right)\right]-\frac{\beta^{2}}{2 \gamma}+\ln 2=I_{0}(m)-\frac{\beta^{2}}{2 \gamma} .
\end{aligned}
$$

Это равняется $I^{\beta}(m)$, если $(\beta, t) \in \Gamma$, т. е. $\beta \leqslant \bar{\beta}(t)$, или $T(\beta) \geqslant t$, так как $\bar{\beta}-$ убывающая функция. Мы покажем, что это действительно происходит в настоящем случае, т. е. при $m \leqslant m(\beta)$. Сначала вычислим

$$
\begin{aligned}
\left(\frac{\partial C_{2}}{\partial t}\right)_{\beta} & =\frac{\partial}{\partial t}\left[\frac{\beta}{\bar{\beta}(\beta, t)} F[\bar{\beta}(\beta, t), \bar{t}(\beta, t)]-\ln 2\right]= \\
& =\frac{\partial}{\partial t}\left[\frac{\beta}{\bar{\beta}} \ln \left[2 \operatorname{ch}\left(\frac{t \bar{\beta}}{\beta}\right)\right]+\frac{\beta \bar{\beta}}{2 \gamma}-\ln 2\right]= \\
& =-\frac{\beta}{\bar{\beta}^{2}} \ln \left[2 \operatorname{ch}\left(\frac{t \bar{\beta}}{\beta}\right)\right] \frac{\partial \bar{\beta}}{\partial t}+\frac{\beta}{\bar{\beta}}\left[\operatorname{th}\left(\frac{t \bar{\beta}}{\beta}\right)\left(\frac{\bar{\beta}}{\beta}+\frac{t}{\beta} \frac{\partial \bar{\beta}}{\partial t}\right)\right]+\frac{\beta}{2 \gamma} \frac{\partial \bar{\beta}}{\partial t}= \\
& =\frac{\beta}{\bar{\beta}^{2}}\left\{-\ln \left[2 \operatorname{ch}\left(\frac{t \bar{\beta}}{\beta}\right)\right]+\frac{t \bar{\beta}}{\beta} \operatorname{th}\left(\frac{t \bar{\beta}}{\beta}\right)+\frac{\bar{\beta}^{2}}{2 \gamma}\right\} \frac{\partial \bar{\beta}}{\partial t}+\operatorname{th}\left(\frac{t \bar{\beta}}{\beta}\right) .
\end{aligned}
$$

Но мы также имеем $t \bar{\beta} / \beta=\bar{t}$, следовательно,

$$
\left(\frac{\partial C_{2}}{\partial t}\right)_{\beta}=\operatorname{th} \bar{t}
$$

Учитывая $C_{2}(\beta, t)$, мы имеем $\bar{\beta} \leqslant \beta$, и это дает $T(\bar{\beta}) \geqslant T(\beta)$. Следовательно,

$$
\left(\frac{\partial C_{2}}{\partial t}\right)_{\beta}=\operatorname{th}[\bar{t}(\beta, t)]=m(\bar{\beta}) \geqslant m(\beta) \geqslant m,
$$

так что $m-\left(\partial C_{2} / \partial t\right)_{\beta}<0$. Это показывает, что максимум функции $I_{2}^{\beta}$ достигается при $t=T(\beta)$, т. е. следует рассматривать $I_{1}^{\beta}$ в этом случае. Следовательно, для $m \leqslant m(\beta)$ мы имеем $I^{\beta}=I_{1}^{\beta}$.

Далее, предположим, что $\beta>\beta_{0}$ или $m \geqslant m(\beta)$. Зная $m$ и $\beta$, мы оцениваем максимайзер функции (21): $t^{*}=\bar{t} \beta / \bar{\beta}$, где $\bar{\beta}$ находится следующим образом. Согласно (26) $m=$ th $\bar{t}$, и мы имеем

$$
\begin{aligned}
\bar{\beta}^{2} & =2 \gamma[\ln [2 \operatorname{ch} \bar{t}]-\bar{t} \operatorname{th} \bar{t}]= \\
& =2 \gamma\left[\ln \left[\operatorname{ch}\left(\operatorname{th}^{-1} m\right)-m \operatorname{th}^{-1} m+\ln 2\right]=2 \gamma\left[\ln 2-I_{0}(m)\right] .\right.
\end{aligned}
$$

Величина $\bar{t}$ неявно дается решением уравнения (28), как только $\bar{\beta}$ найдено для данного $m$. Итак, мы нашли максимайзер $t^{*}$ правой части соотношения $(21)$, теперь вычислим $I_{2}^{\beta}(m)$, используя выражение $(28)$ для $\bar{\beta}^{2}$ :

$$
\begin{aligned}
I_{2}^{\beta}(m) & =t^{*} m-C_{2}\left(\beta, t^{*}\right)=\frac{\beta}{\bar{\beta}} \bar{t} m-\frac{\beta}{\bar{\beta}} \ln [2 \operatorname{ch} \bar{t}]-\frac{\bar{\beta} \beta}{2 \gamma}+\ln 2= \\
& =\frac{\beta}{2 \gamma \bar{\beta}} 2 \gamma\left(I_{0}(m)-\ln 2\right)-\frac{\beta \bar{\beta}}{2 \gamma}+\ln 2=-\frac{\beta \bar{\beta}}{\gamma}+\ln 2 .
\end{aligned}
$$




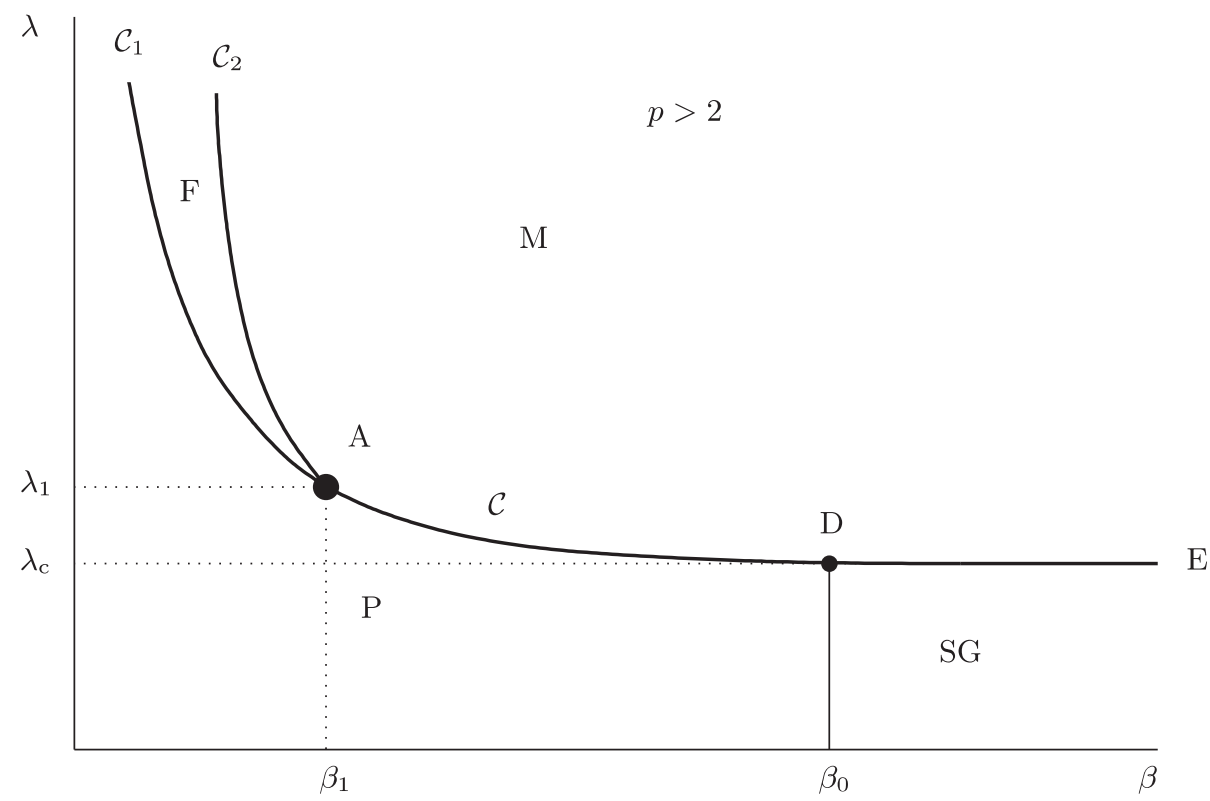

Рис. 2. Фазовая диаграмма. В парамагнитной области (Р) $m=0$, но свободная энергия зависит от $\beta$; а в ферромагнитной области $(\mathrm{F}) m(\beta, \lambda) \neq 0$, в то время как в фазе спинового стекла (SG) $m=0$ и свободная энергия остается постоянной. В смешанной фазе $(\mathrm{M}) m \neq 0$ и свободная энергия зависит только от $\lambda$. Магнетизация $m$ прерывается в направлении поперек кривых $\mathcal{C}_{1}, \mathcal{C}$ и $\lambda=\lambda_{\mathrm{c}}$, в то время как она является непрерывной в направлении поперек кривой $\mathcal{C}_{2}$. Мы ожидаем, что упорядоченная ферромагнитная фаза уменьшится в высокотемпературном режиме в случае больших $p$, что доказывается в разделе 5 .

Это равенство справедливо всякий раз, когда $\beta \geqslant \bar{\beta}(t)$, или, что эквивалентно, когда $m \geqslant m(\beta)$.

Покажем, что в этом случае, т. е. когда $m \geqslant m(\beta)$, ни одно значение $t$ не может быть максимайзером величины $I_{1}^{\beta}$ : неравенство

$$
m-\frac{\partial C_{1}}{\partial t}=m-\operatorname{th} t \geqslant 0,
$$

вместе с $C_{1} t \leqslant T(\beta)$ влечет th $t \leqslant m(\beta)$. Следовательно, если $m \geqslant m(\beta)$, то $I^{\beta}=I_{2}^{\beta}$. И также если $m \geqslant 1$, то ясно, что $I^{\beta}(m)=\infty$.

\section{4. СВОБОДНАЯ ЭНЕРГИЯ И ФАЗОВАЯ ДИАГРАММА $(p>2)$}

В этом разделе мы представим основные результаты настоящей статьи, которые сформулированы и доказаны в теореме 2 .

Сначала определим кривые $\lambda=\mathcal{C}_{1}(\beta), \lambda=\mathcal{C}_{2}(\beta)$ :

$$
\mathcal{C}_{1}(\beta)=\frac{1}{2 \beta}, \quad \mathcal{C}_{2}(\beta)=\frac{T(\beta)}{2 \beta m(\beta)}
$$




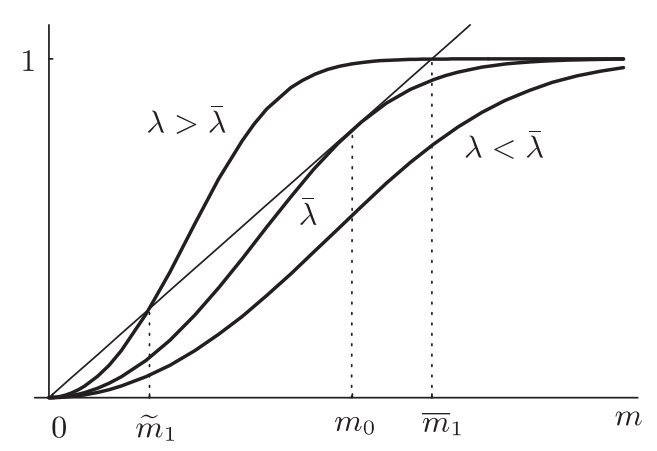

a

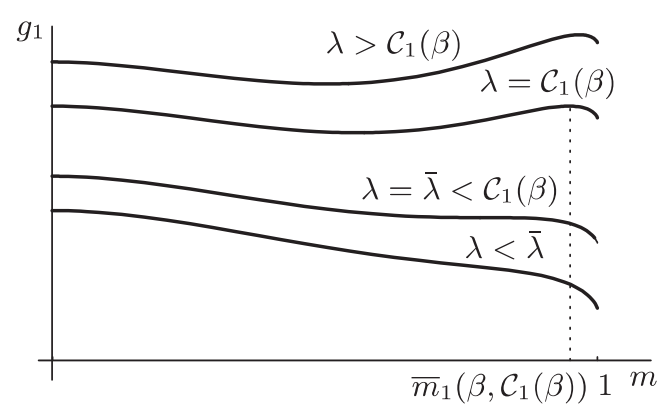

б

Рис. 3. Решения уравнения (36): $\operatorname{th}\left[p \beta \lambda m^{p-1}\right]$ при $\lambda>\tilde{\lambda}, \lambda=\tilde{\lambda}$ и $\lambda<\tilde{\lambda}$ (а); $\lambda=\mathcal{C}_{1}(\beta)$ определяет критическое значение (б).

(см. рис. 2), где $m(\beta)=\operatorname{th}[T(\beta)]$. Также определим

$$
G_{1}(m)=\left[1+\left(1-\frac{1}{p}\right) m\right] \ln (1+m)+\left[1-\left(1-\frac{1}{p}\right) m\right] \ln (1-m)
$$

и

$$
g_{i}(\beta, \lambda ; m)=\beta \lambda m^{p}-I_{i}^{\beta}(m), \quad i=1,2 .
$$

ЛЕмма 1. Пусть $\beta<\beta_{0}, u$ мы определим

$$
F_{1}(\beta, \lambda)=\sup _{0 \leqslant m \leqslant m(\beta)}\left\{\beta \lambda m^{p}-I_{1}^{\beta}(m)\right\}
$$

где $m(\beta)=\operatorname{th}[T(\beta)]$. Тогда происходят скачки магнетизации от 0 до $m_{p}$ поперек кривой $\lambda=\mathcal{C}_{1}(\beta)$, где $m_{p}$ не зависит от $\beta, \lambda$ и дается решением уравнения $G_{1}(m)=0$.

ДокАЗАТЕЛЬство. Ясно, что супремум выражения (34) достигается, если $m$ удовлетворяет уравнению

$$
m=\operatorname{th}\left[p \beta \lambda m^{p-1}\right]
$$

в случае решения $\bar{m} \leqslant m(\beta)$. Действительно, при фиксированном $\beta$ имеется критическое значение $\tilde{\lambda}(\beta)$, ниже которого уравнение (35) имеет только нулевое решение. Тогда при $\lambda \leqslant \tilde{\lambda}(\beta)$ мы имеем $\partial g_{1} / \partial m \leqslant 0$, откуда получаем

$$
F_{1}(\beta, \lambda)=-I_{1}^{\beta}(0)
$$

При $\lambda>\tilde{\lambda}(\beta)$ уравнение (36) имеет ненулевые решения $\widetilde{m}_{1}(\beta, \lambda)$ и $\bar{m}_{1}(\beta, \lambda)>$ $\widetilde{m}_{1}(\beta, \lambda)$ (см. рис. 3a).

Ясно, что функция $g_{1}$ имеет локальный минимум при $\widetilde{m}_{1}$ и локальный максимум при $\bar{m}_{1}$ (см. рис. За, 3б), однако неверно считать, что при этом $\tilde{\lambda}$ будет критическим значением $\lambda$. Действительно,

$$
g_{1}\left(\beta, \tilde{\lambda} ; \bar{m}_{1}\right)<g_{1}(\beta, \tilde{\lambda} ; 0),
$$

5 Теоретическая и математическая физика, т. 166, № 1, 2011 г. 


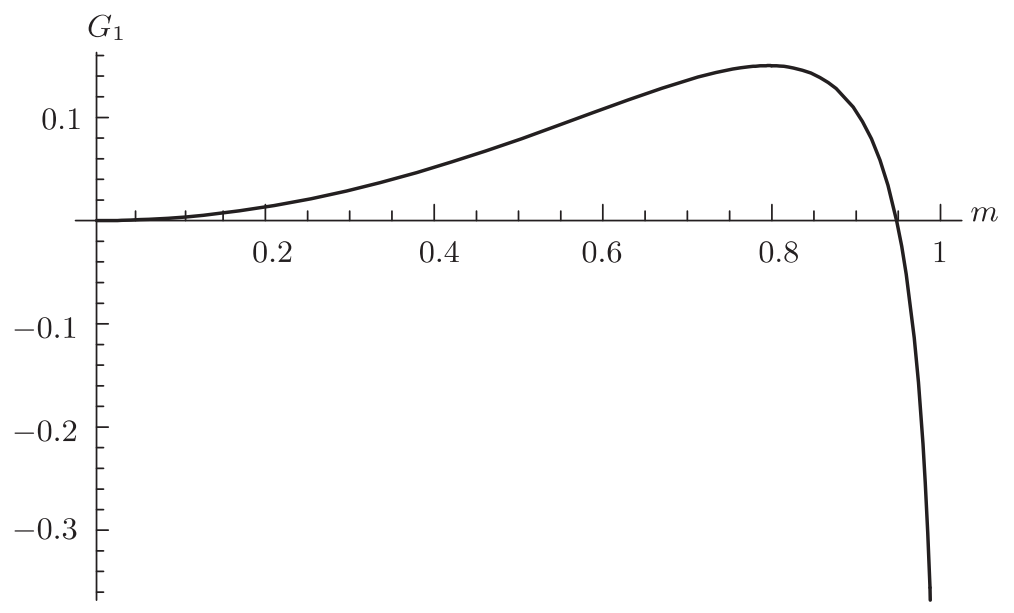

Рис. 4. Кривая $G_{1}(m)=0$ имеет единственное решение на отрезке $(0,1)$.

из-за непрерывности так и будет, если $\lambda$ возрастает не сильно. Магнетизация станет ненулевой, когда $\lambda$ достигнет значения, при котором

$$
g_{1}\left(\beta, \lambda ; \bar{m}_{1}\right)=g_{1}(\beta, \lambda ; 0)=-I_{1}^{\beta}(0) .
$$

Это определяет критическую линию $\lambda=\mathcal{C}_{1}(\beta)$ (см. рис. 3б). Поперек линии $\lambda=$ $\mathcal{C}_{1}(\beta)$ магнетизация изменяется скачком от нуля до значения $m_{p}$, задаваемого положительным решением уравнения (35) таким, что

$$
-I_{1}^{\beta}(0)=\beta \lambda m_{p}^{p}-I_{1}^{\beta}\left(m_{p}\right) \quad \Rightarrow \quad \beta \lambda m_{p}^{p}=I_{0}\left(m_{p}\right) .
$$

В совокупности с $(36)$ это дает $G_{1}\left(m_{p}\right)=0$.

ЛЕмма 2. Для любого $p>2$ уравнение $G_{1}(m)=0$ имеет единственное решение $m_{p} \in(0,1)$. Более того, при больиих р справедливо $m_{p} \sim 1-2^{-(2 p-1)}$.

ДоКАЗАТЕЛЬСТво. Мы имеем $G_{1}(0)=0, G_{1}(1)=-\infty$ и

$$
\begin{aligned}
G_{1}^{\prime}(m) & =\left(1-\frac{1}{p}\right) \ln \frac{1+m}{1-m}+\frac{1+(1-1 / p) m}{1+m}-\frac{1-(1-1 / p) m}{1-m} \\
G_{1}^{\prime}(0) & =0, \quad G_{1}^{\prime}(1)=-\infty
\end{aligned}
$$

Также мы находим

$$
G_{1}^{\prime \prime}(m)=\frac{2}{1-m^{2}}\left[1-\frac{1}{p}-\frac{1}{p} \frac{1+m^{2}}{1-m^{2}}\right], \quad G_{1}^{\prime \prime}(0)=2\left(1-\frac{2}{p}\right)>0 .
$$

Так как $\left(1+m^{2}\right) /\left(1-m^{2}\right)$ увеличивается от 1 до $+\infty$ при возрастании $m$ от 0 до 1 , то имеется единственное значение $m_{1}>0$ такое, что $G_{1}^{\prime \prime}\left(m_{1}\right)=0$, и $G_{1}^{\prime \prime}(m)>0$ при $m<m_{1}$ и $G_{1}^{\prime \prime}(m)<0$ при $m>m_{1}$. Следовательно, функция $G_{1}^{\prime}(m)$ является возрастающей от $m=0$ до $m=m_{1}$ и убывающей от $m=m_{1}$ до $m=1$. 
Так как $G_{1}^{\prime}(0)=0, G_{1}^{\prime}\left(m_{1}\right)>0$ и $G_{1}^{\prime}(1)=-\infty$, то имеется единственное значение $m_{2}>m_{1}$ такое, что $G_{1}^{\prime}\left(m_{2}\right)=0$. Таким образом, функция $G_{1}$ является возрастающей от $m=0$ до $m=m_{2}$ и убывающей при $m>m_{2}$. Тогда имеется единственное значение $m_{p}>m_{2}$ такое, что $G_{1}\left(m_{p}\right)=0$. Форма кривой $G_{1}$ показана на рис. 4 . Чтобы завершить доказательство леммы, мы положим $m=1-\delta$. Ясно, что при больших $p$

$$
\begin{aligned}
\ln \delta & =-\frac{1+(1-1 / p) m}{1-(1-1 / p) m} \ln (1+m) \sim-\frac{2-1 / p}{1 / p} \ln 2= \\
& =-(2 p-1) \ln 2 \Rightarrow \delta \sim 2^{-(2 p-1)} .
\end{aligned}
$$

ЛЕмма 3. Выберем $\tilde{\lambda}(\beta)$ как в лемме 1 , и пусть $m_{0}>0$ будет таким значением, при котором $m_{0}=\operatorname{th}\left[\beta \tilde{\lambda} p m_{0}^{p-1}\right]$ (см. рис. За), а значение $\beta_{\max }$ будет таким, что $m\left(\beta_{\max }\right)=m_{0}$.

При $\beta<\beta_{\max }$ справедливо $m(\beta)>m_{0}$, тогда имеется единственная кривая $\lambda=\mathcal{C}_{2}(\beta)$, для которой $\bar{m}_{1}(\beta, \lambda)=m(\beta)$, и только эта часть кривой $\lambda=\mathcal{C}_{2}(\beta)$ является существенной в фазовой диаграмме.

ДокАЗАтЕЛЬство. Если подставить $m_{p}$ в уравнение (35), получим

$$
\mathcal{C}_{1}(\beta)=\frac{\operatorname{th}^{-1} m_{p}}{p \beta m p^{p-1}} .
$$

При $\lambda>\mathcal{C}_{1}(\beta)$ максимум достигается при $m=\bar{m}_{1} \geqslant m_{p}$, если $\bar{m}_{1} \leqslant m(\beta)$. Таким образом, мы получаем еще одно критическое значение $\lambda$, выше которого $m=m(\beta)$. Эта кривая дается уравнением $\lambda=\mathcal{C}_{2}(\beta)$, где

$$
\mathcal{C}_{2}(\beta)=\frac{\operatorname{th}^{-1}(m(\beta))}{p \beta(m(\beta))^{p-1}} .
$$

Заметим, что $m(\beta) \rightarrow 1$ при $\beta \rightarrow 0$, так что для малых, но возрастающих $\beta$, мы имеем $\mathcal{C}_{2}(\beta)>\mathcal{C}_{1}(\beta)$, и $m(\beta)$ уменьшается до тех пор, пока не достигнет $m_{p}$.

Определим $\beta_{1}$ :

$$
m\left(\beta_{1}\right)=m_{p} .
$$

Тогда $\mathcal{C}_{1}\left(\beta_{1}\right)=\mathcal{C}_{2}\left(\beta_{1}\right)$. Имеется минимальное значение $\lambda_{\min }$, ниже которого уравнение

$$
m=m(\beta), \quad m=\bar{m}_{1}(\beta, \lambda)
$$

не имеет решения. В случае $\lambda<\tilde{\lambda}\left(\beta_{\max }\right)=\lambda_{\min }$ мы должны увеличить $\beta$, чтобы уравнение (35) имело решение, но тогда функция $m(\beta)$ становится меньше $m_{0}$ и недействительна. Так как $\beta<\beta_{\max }, m(\beta)>m_{0}$, то имеется единственное значение $\lambda=\mathcal{C}_{2}(\beta)$, при котором $\bar{m}_{1}(\beta, \lambda)=m(\beta)$; отсюда следует, что только эта часть кривой $\lambda=\mathcal{C}_{2}(\beta)$ является существенной.

Лемма 4. Для $\beta>\beta_{1}$ справедливо $\bar{m}_{1}(\beta, \lambda)>m(\beta)$ и существует критическая кривая на фазовой диаграмме, задаваемая уравнением

$$
\mathcal{C}_{3}(\beta)=\frac{\beta_{0}^{2}-\beta^{2}}{2 \gamma \beta m^{p}(\beta)} .
$$

Более того, $\mathcal{C}_{3}(\beta) \rightarrow+\infty$ при $\beta \rightarrow \beta_{0}$. 
ДокАЗАТЕльСтво. Заметим, что $\tilde{\lambda}(\beta)=c_{0} / \beta$ (для некоторой постоянной $c_{0}$ ), так как $\bar{m}_{1}$ зависит только от $\beta \lambda$. При $\beta \lambda<c_{0}$ уравнение (35) не имеет решений, в то время как при $c>c_{0}$ гипербола $\beta \lambda=c$ пересекает кривую $\lambda=\mathcal{C}_{2}(\beta)$ в двух точках: одна из них с $\beta<\beta_{\max }$ соответствует $m(\beta)=\bar{m}_{1}(\beta, \lambda)$, и другая с $\beta>\beta_{\max }-$ $m(\beta)=\widetilde{m}_{1}(\beta, \lambda)$.

Отсюда следует, что для $\beta>\beta_{1}$ имеет место $\bar{m}_{1}(\beta, \lambda)>m(\beta)$. Итак, мы должны сравнить $-I_{1}^{\beta}(0)$ и $\beta \lambda m^{p}(\beta)-I_{1}^{\beta}(m(\beta))$. Это определяет критическую линию $\mathcal{C}_{3}(\beta)$ :

$$
\lambda=\frac{I_{1}^{\beta}(m(\beta))-I_{1}^{\beta}(0)}{\beta m^{p}(\beta)}=\frac{I_{0}(m(\beta))}{\beta m^{p}(\beta)} \equiv \mathcal{C}_{3}(\beta) .
$$

Заметим, что

$$
\begin{aligned}
I_{0}(m(\beta)) & =\frac{1}{2} \ln \left(1-m^{2}(\beta)\right)+m(\beta) \operatorname{th}^{-1} m(\beta)= \\
& =\frac{1}{2} \ln \frac{1}{\operatorname{ch}^{2}(T(\beta))}+T(\beta) \operatorname{th}[T(\beta)]= \\
& =-\ln [\operatorname{ch}(T(\beta))]+T(\beta) \operatorname{th}[T(\beta)]= \\
& =-\frac{\bar{\beta}(T(\beta))^{2}}{2 \gamma}+\ln 2=\frac{\beta_{0}^{2}-\beta^{2}}{2 \gamma} .
\end{aligned}
$$

Итак, мы имеем $\mathcal{C}_{3}(\beta)=\left(\beta_{0}^{2}-\beta^{2}\right) / 2 \gamma \beta m^{p}(\beta)$, что и требовалось доказать. При $\beta \rightarrow \beta_{0}$ получаем $m(\beta) \rightarrow 0$, а более точно

$$
\begin{aligned}
\bar{\beta}(t) & \sim\left\{2 \gamma\left(\ln 2-\frac{t^{2}}{2}\right)\right\}^{1 / 2} \sim \beta_{0}\left(1-\frac{t^{2}}{4 \ln 2}\right) \Rightarrow \\
& \Rightarrow T(\beta) \sim\left\{\frac{4 \ln 2}{\beta_{0}}\left(\beta_{0}-\beta\right)\right\}^{1 / 2} \Rightarrow m(\beta) \sim\left\{\frac{4 \ln 2}{\beta_{0}}\left(\beta_{0}-\beta\right)\right\}^{1 / 2} .
\end{aligned}
$$

Отсюда получаем, что $\mathcal{C}_{3}(\beta) \rightarrow+\infty$ при $\beta \rightarrow \beta_{0}$.

Теперь можно сформулировать следующую лемму.

Лемма 5. Пусть $\beta_{1}-$ точка пересечения кривых $\mathcal{C}_{1}(\beta)$ и $\mathcal{C}_{2}(\beta)$. Если $0<\beta \leqslant \beta_{1}$, mo

$$
F_{1}(\beta, \lambda)= \begin{cases}-I_{1}^{\beta}(0), & \lambda \leqslant \mathcal{C}_{1}(\beta), \\ \beta \lambda \bar{m}_{1}^{p}-I_{1}^{\beta}\left(\bar{m}_{1}\right), & \mathcal{C}_{1}(\beta) \leqslant \lambda \leqslant \mathcal{C}_{2}(\beta), \\ \beta \lambda m^{p}(\beta)-I_{1}^{\beta}(m(\beta)), & \lambda>\mathcal{C}_{2}(\beta) .\end{cases}
$$

Если $\beta_{1}<\beta<\beta_{0}$, mо

$$
F_{1}(\beta, \lambda)= \begin{cases}-I_{1}^{\beta}(0), & \lambda \leqslant \mathcal{C}_{3}(\beta), \\ \beta \lambda m^{p}(\beta)-I_{1}^{\beta}(m(\beta)), & \lambda>\mathcal{C}_{3}(\beta) .\end{cases}
$$

ДокаЗАтЕЛЬСтво. Остается только доказать, что при $\lambda>\mathcal{C}_{2}(\beta)$ мы имеем $F_{1}(\beta, \lambda)=\beta \lambda m^{p}(\beta)-I_{1}^{\beta}(m(\beta))$. Это следует из того факта, что $\mathcal{C}_{3}(\beta)<\mathcal{C}_{2}(\beta)$ при $\beta<\beta_{1}$. Но это эквивалентно $G_{1}(m(\beta))<0$, и согласно доказательству леммы 2 это эквивалентно $m(\beta)>m_{p}$, т. е. $\beta<\beta_{1}$. 
Лемма 6. Определим

$$
F_{2}(\beta, \lambda):=\sup _{m \geqslant m(\beta)}\left\{\beta \lambda m^{p}-I_{2}^{\beta}(m)\right\} .
$$

Тогда существует такое значение $\lambda_{2}>0$, что для всех $\lambda<\lambda_{2}$ функция $F_{2}$ имеет глобальный максимайзер, скажем $\bar{m}_{2}(\lambda)$, при достаточно больших $\lambda$. Функиия $F_{2}$ имеет нуль в качестве максимайзера при $\lambda \geqslant \lambda_{2}$.

ДокАЗАТЕЛЬство. Условие максимизации функции (49) имеет вид

$$
\begin{aligned}
p \beta \lambda m^{p-1} & =\frac{d}{d m} I_{2}^{\beta}(m)=\frac{d}{d m}\left[\ln 2-\frac{\beta}{\gamma}\left[\beta_{0}^{2}-2 \gamma I_{0}(m)\right]^{1 / 2}\right]= \\
& =\frac{\beta}{\bar{\beta}} \operatorname{th}^{-1} m \quad \Rightarrow \quad m=\operatorname{th}\left[p \bar{\beta}(m) \lambda m^{p-1}\right] .
\end{aligned}
$$

Теперь докажем, что $\operatorname{th}\left[p \bar{\beta} \lambda m^{p-1}\right]$ имеет в точности одну поворотную точку на отрезке $(0,1)$. Находим

$$
\begin{aligned}
& \frac{d}{d m} \operatorname{th}\left[p \bar{\beta} \lambda m^{p-1}\right]=\operatorname{sch}^{2}\left(p \bar{\beta} \lambda m^{p-1}\right)\left[p \lambda(p-1) m^{p-2} \bar{\beta}+p \lambda m^{p-1} \bar{\beta}^{\prime}\right]= \\
& \quad=p \lambda m^{p-2} \operatorname{sch}^{2}\left(p \bar{\beta} \lambda m^{p-1}\right)\left[(p-1) \bar{\beta}+m \bar{\beta}^{\prime}\right]
\end{aligned}
$$

И

$$
\bar{\beta}^{\prime}=-\frac{\gamma \operatorname{th}^{-1} m}{\bar{\beta}} .
$$

Подставляя приведенное выше выражение в (50), мы получим $d\left[\operatorname{th}\left(p \bar{\beta} \lambda m^{p-1}\right] / d m=\right.$ 0 тогда и только тогда, когда

$$
(p-1) \bar{\beta}=\frac{m \gamma \operatorname{th}^{-1} m}{\bar{\beta}},
$$

т. e.

$$
2(p-1) I_{0}(m)+m \text { th }^{-1} m-2(p-1) \ln 2=0 .
$$

Так как обе функции $I_{0}(m)$ и $m \mathrm{th}^{-1} m$ являются выпуклыми и возрастающими и обе равны нулю при $m=0$, уравнение (52) всегда имеет в точности одно ненулевое решение, которое показывает, что функция $\operatorname{th}\left[p \bar{\beta} \lambda m^{p-1}\right]$ имеет в точности одну поворотную точку на отрезке $[0,1]$. Так как функция th $\left[p \bar{\beta} \lambda m^{p-1}\right]=0$ при $m=0,1$ и является неотрицательной величиной для $m \in[0,1]$, ясно, что приведенный выше экстремум является максимумом. Изучая кривые $y=m^{p-1}$ и $y=\bar{\beta}(m)=$ $\left\{\beta_{0}^{2}-2 \gamma I_{0}(m)\right\}^{1 / 2}$, мы видим, что функция $\operatorname{th}\left[p \bar{\beta} \lambda m^{p-1}\right]$ не является ни выпуклой, ни вогнутой. В действительности она является выпуклой на $\left(0, m_{1}\right)$ и вогнутой на $\left(m_{1}, 1\right)$ для некоторых $m_{1} \in(0,1)$; так что уравнение $m=\operatorname{th}\left[p \bar{\beta} \lambda m^{p-1}\right]$ должно иметь не более двух ненулевых решений (см. рис. 5$)$. Пусть $\widetilde{m}_{2}, \bar{m}_{2} \in(0,1), \widetilde{m}_{2}<\bar{m}_{2}$, будут этими двумя решениями. Из формы кривой $y=\operatorname{th}\left[p \bar{\beta} \lambda m^{p-1}\right]$ следует

$$
\begin{array}{lll}
m \geqslant \operatorname{th}\left[p \bar{\beta} \lambda m^{p-1}\right], & \text { если } \quad m \leqslant \widetilde{m}_{2} \quad \text { или } \quad m \geqslant \bar{m}_{2}, \\
m \leqslant \operatorname{th}\left[p \bar{\beta} \lambda m^{p-1}\right], & \text { если } \quad \widetilde{m}_{2} \leqslant m \leqslant \bar{m}_{2} .
\end{array}
$$




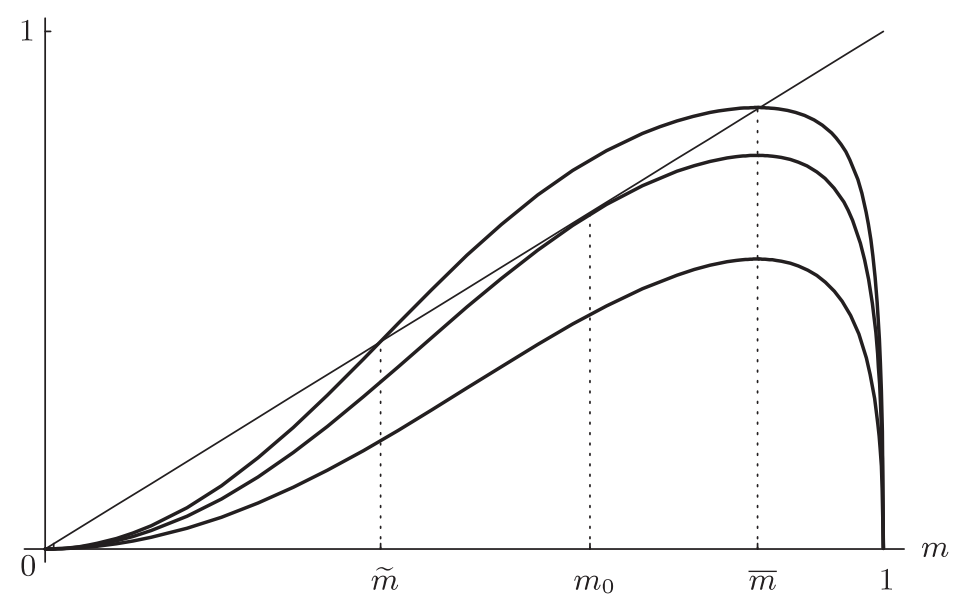

Рис. 5. Функция $m=\operatorname{th}\left[p \bar{\beta} \lambda m^{p-1}\right]$ имеет два ненулевых решения $\widetilde{m}_{2}, \bar{m}_{2}$; $\widetilde{m}_{2}$ уменьшается и $\bar{m}_{2}$ увеличивается при увеличении $\lambda$.

Так как функция $\operatorname{th}\left[p \bar{\beta} \lambda m^{p-1}\right]$ является возрастающей по $\lambda$, то ясно, что $\widetilde{m}_{2}$ уменьшается, а $\bar{m}_{2}$ увеличивается с увеличением $\lambda$.

Как и в случае уравнения (35), существует такое значение $\lambda_{2}>0$, что уравнение (49) имеет только нулевое решение, если $\lambda \geqslant \lambda_{2}$ (однако $\lambda_{2}$ не зависит от $\beta$ ). Для $\lambda<\lambda_{2}$ существуют два решения, а именно $\widetilde{m}_{2}(\lambda)$ и $\bar{m}_{2}(\lambda)$. Функция $g_{2}(\beta, \lambda ; m)$ имеет локальный максимум при наибольшем из них, т. е. при $\bar{m}_{2}(\lambda)$. И опять он может быть только глобальным максимумом, если $\lambda$ достаточно велико, т. е.

$$
\beta \lambda \bar{m}_{2}^{p}(\lambda)-I_{2}^{\beta}\left(\bar{m}_{2}(\lambda)\right) \geqslant \beta \lambda m^{p}(\beta)-I_{2}^{\beta}(m(\beta)) .
$$

Итак, мы можем написать $\beta_{2}=\bar{\beta}\left(\lambda_{2}\right)$, т. е. $\lambda_{2}=m\left(\beta_{2}\right)$.

Справедливо следующее утверждение.

Лемма 7. Для $\beta \leqslant \beta_{2}$ неравенство (54) эквивалентно $\lambda \geqslant \mathcal{C}_{2}(\beta)$.

ДокАЗАтельство. Пусть $\bar{m}_{2}(\lambda)=m(\beta)=m$. Тогда $\bar{\beta}(m)=\beta$, т. е. (49) эквивалентно (35). Таким образом, мы имеем случай, аналогичный (41): горизонтальные линии $\lambda=$ const $>\lambda_{2}$ пересекают $\lambda=\mathcal{C}_{2}(\beta)$ в двух точках; одна из них с $\beta<\beta_{2}$ соответствует $m(\beta)=\bar{m}_{2}(\lambda)$, а другая с $\beta>\beta_{2}-m(\beta)=\widetilde{m}_{2}(\lambda)$. Теперь пусть $\lambda \geqslant \mathcal{C}_{2}(\beta)$. Тогда $\bar{m}_{2}(\lambda) \geqslant m(\beta) \geqslant \widetilde{m}_{2}(\lambda)$, т. е. $g_{2}(\beta, \lambda ; m)$ возрастает от $m(\beta)$ до $\bar{m}_{2}(\lambda)$. И наоборот, пусть $\lambda<\mathcal{C}_{2}(\beta)$. Тогда $\bar{m}_{2}(\lambda)<m(\beta)$, и решение не является действительным.

ЗАмечАниЕ. Для $\beta_{2}<\beta<\beta_{0}$ неравенство (54) определяет кривую $\lambda=\mathcal{C}_{4}(\beta)$, которая не совпадает с $\lambda=\mathcal{C}_{2}(\beta)$. Прежде чем обсуждать этот случай, мы рассмотрим случай $\beta>\beta_{0}$.

Лемма 8. Пусть $\beta>\beta_{0}$, определим функцию

$$
G_{2}(m):=\frac{p}{\gamma} \bar{\beta}(m)\left(\beta_{0}-\bar{\beta}(m)\right)-m \operatorname{th}^{-1} m .
$$


Пусть $p>2$. Тогда уравнение $G_{2}(m)=0$ имеет единственное решение $m=m_{c} \in$ $(0,1)$ и, более того, для больших $p$

$$
1-m_{\mathrm{c}} \sim \frac{1}{8 p^{2} \ln 2} \ln \left(8 p^{2} \ln 2\right) .
$$

ДокАЗАТЕЛЬСтво. Если $\lambda<\lambda_{2}$, то $g_{2}(\beta, \lambda ; m)$ является убывающей функцией; тогда

$$
F_{2}(\beta, \lambda)=-I_{2}^{\beta}(0)
$$

Для $\lambda>\lambda_{2}$ мы должны сравнить $-I_{2}^{\beta}(0)$ и $\beta \lambda \bar{m}_{2}^{p}(\lambda)-I_{2}^{\beta}\left(\bar{m}_{2}(\lambda)\right)$. Критическое зна-

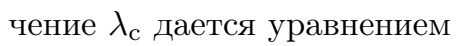

$$
-I_{2}^{\beta}(0)=\beta \lambda \bar{m}_{2}^{p}\left(\lambda_{\mathrm{c}}\right)-I_{2}^{\beta}\left(\bar{m}_{2}\left(\lambda_{\mathrm{c}}\right)\right)
$$

Заметим, что это выражение не зависит от $\beta>\beta_{0}$ :

$$
\lambda_{\mathrm{c}}=\frac{\beta_{0}-\bar{\beta}\left(m_{\mathrm{c}}\right)}{\gamma m_{\mathrm{c}}^{p}},
$$

где $m_{\mathrm{c}}=\bar{m}_{2}\left(\lambda_{\mathrm{c}}\right)$. Подставляя (59) в (49) с $\lambda=\lambda_{\mathrm{c}}$, мы имеем $G_{2}\left(m_{\mathrm{c}}\right)=0$, где

$$
G_{2}(m)=\frac{p}{\gamma} \bar{\beta}(m)\left(\beta_{0}-\bar{\beta}(m)\right)-m \mathrm{th}^{-1} m .
$$

Покажем, что функция $G_{2}(m)$ имеет ту же форму, что и $G_{1}(m)$ (см. доказательство леммы 2). Сначала вычислим

$$
G_{2}^{\prime}(m)=\frac{p}{\gamma} \bar{\beta}^{\prime}(m)\left(\beta_{0}-2 \bar{\beta}(m)\right)-\mathrm{th}^{-1} m-\frac{m}{1-m^{2}}
$$

и

$$
G_{2}^{\prime \prime}(m)=\frac{p}{\gamma} \bar{\beta}^{\prime \prime}(m)\left(\beta_{0}-2 \bar{\beta}(m)\right)-\frac{2 p}{\gamma} \bar{\beta}^{\prime 2}(m)-\frac{2}{\left(1-m^{2}\right)^{2}} .
$$

Используя соотношения

$$
\bar{\beta}^{\prime}(m)=-\gamma \frac{\operatorname{th}^{-1} m}{\bar{\beta}(m)}
$$

и

$$
\bar{\beta}^{\prime \prime}(m)=-\frac{\gamma}{\left(1-m^{2}\right) \bar{\beta}(m)}-\frac{\gamma^{2}\left(\mathrm{th}^{-1} m\right)^{2}}{\bar{\beta}^{3}(m)}=-\frac{\gamma}{\left(1-m^{2}\right) \bar{\beta}(m)}-\frac{\bar{\beta}^{\prime 2}(m)}{\bar{\beta}(m)},
$$

мы получаем

$$
\begin{aligned}
G_{2}^{\prime \prime}(m) & =-\frac{p \beta_{0}}{\bar{\beta}(m)}\left(\frac{1}{1-m^{2}}+\frac{\bar{\beta}^{\prime 2}(m)}{\gamma}\right)+\frac{2 p}{1-m^{2}}-\frac{2}{\left(1-m^{2}\right)^{2}}= \\
& =\frac{1}{1-m^{2}}\left\{p\left(2-\frac{\beta_{0}}{\bar{\beta}(m)}\right)-\frac{2}{1-m^{2}}-\frac{p}{\gamma} \frac{\beta_{0}}{\bar{\beta}(m)}\left(1-m^{2}\right) \bar{\beta}^{\prime 2}(m)\right\} .
\end{aligned}
$$

Как и в доказательстве леммы 2, мы покажем, что выражение в фигурных скобках является убывающим по $m$. Согласно $(63) \bar{\beta}(m)$ является убывающей функцией, 
так что первый член является убывающим. Второй член, очевидно, является убывающим. Так как $\beta_{0} / \bar{\beta}(m)$ - возрастающая функция, то достаточно показать, что

$$
\frac{d\left[\left(1-m^{2}\right) \bar{\beta}^{\prime 2}(m)\right]}{d m} \geqslant 0 .
$$

Теперь мы имеем

$$
\frac{d}{d m}\left[\left(1-m^{2}\right) \bar{\beta}^{\prime 2}(m)\right]=2 \bar{\beta}^{\prime}(m)\left[\left(1-m^{2}\right) \bar{\beta}^{\prime \prime}(m)-m \bar{\beta}^{\prime}(m)\right]=-2 \frac{\bar{\beta}^{\prime}(m)}{\bar{\beta}^{3}(m)} \rho(m),
$$

где

$$
\rho(m)=\gamma^{2}\left(1-m^{2}\right)\left(\mathrm{th}^{-1} m\right)^{2}+\gamma\left(1-m \mathrm{th}^{-1} m\right) \bar{\beta}^{2}(m) .
$$

Но $\rho(m) \rightarrow 0$ при $m \rightarrow 1$,

$$
\begin{aligned}
\rho^{\prime}(m)= & -2 m \gamma^{2}\left(\operatorname{th}^{-1} m\right)^{2}+2 \gamma^{2} \operatorname{th}^{-1} m-\gamma \bar{\beta}^{2}(m) \operatorname{th}^{-1} m-\frac{m}{1-m^{2}} \bar{\beta}^{2}(m)- \\
& -2 \gamma^{2} \operatorname{th}^{-1} m\left(1-m \mathrm{th}^{-1} m\right)<0 .
\end{aligned}
$$

Отсюда следует, что $\rho(m)>0$, и поскольку $\bar{\beta}^{\prime}(m)<0$, то $d\left[\left(1-m^{2}\right) \bar{\beta}^{\prime 2}(m)\right] / d m \geqslant 0$. Таким образом, выражение в фигурных скобках в (65) является убывающим. При $m=0$ оно равняется $p-2>0$, и при $m \rightarrow 1$ оно стремится к $-\infty$. Следовательно, кривая $G_{2}^{\prime \prime}(m)$ имеет единственный нуль. Оставшаяся часть доказательства единственности и существования такая же, как в лемме 2.

Ясно, что при больших $p$ значение $m_{\mathrm{c}}$ должно быть близким к единице. Однако асимптотически при $m \sim 1$

$$
\bar{\beta}(m) \sim[\gamma(1-m)|\ln (1-m)|]^{1 / 2}
$$

и $m \mathrm{th}^{-1} m \sim(\ln 2-\ln (1-m)) / 2$, так что

$$
p[2 \ln 2(1-m)|\ln (1-m)|]^{1 / 2} \sim \frac{1}{2}|\ln (1-m)| .
$$

Отсюда следует, что

$$
\frac{1-m}{|\ln (1-m)|} \sim \frac{1}{8 p^{2} \ln 2} \quad \Rightarrow \quad 1-m \sim \frac{1}{8 p^{2} \ln 2} \ln \left(8 p^{2} \ln 2\right) .
$$

Лемма 9. Пусть $\beta_{2}$ - минимум кривой $\mathcal{C}_{2}(\beta)$, задаваемой уравнением (39). Тогда $\partial л я 0<\beta \leqslant \beta_{2}$

$$
F_{2}(\beta, \lambda)= \begin{cases}\beta \lambda m^{p}(\beta)-I_{2}^{\beta}(m(\beta)), & \lambda \leqslant \mathcal{C}_{2}(\beta), \\ \beta \lambda \bar{m}_{2}^{p}(\lambda)-I_{2}^{\beta}\left(\bar{m}_{2}(\lambda)\right), & \lambda>\mathcal{C}_{2}(\beta),\end{cases}
$$

a $\partial \Omega \Omega \beta \beta_{0}$

$$
F_{2}(\beta, \lambda)= \begin{cases}-I_{2}^{\beta}(0), & \lambda \leqslant \lambda_{c}, \\ \beta \lambda \bar{m}_{2}^{p}(\lambda)-I_{2}^{\beta}\left(\bar{m}_{2}(\lambda)\right), & \lambda \geqslant \lambda_{\mathrm{c}} .\end{cases}
$$

Наконеи, для $\beta_{2} \leqslant \beta \leqslant \beta_{0}$ существует кривая $\lambda=\mathcal{C}_{4}(\beta)$, задаваемая уравнением (54), лежащая ниже $\lambda=\mathcal{C}_{2}(\beta)$ и удовлетворлющая $\mathcal{C}_{4}\left(\beta_{2}\right)=\lambda_{2}$ и $\mathcal{C}_{4}\left(\beta_{0}\right)=\lambda_{\mathrm{c}}$, mak чmo

$$
F_{2}(\beta, \lambda)= \begin{cases}\beta \lambda m^{p}(\beta)-I_{2}^{\beta}(m(\beta)), & \lambda \leqslant \mathcal{C}_{4}(\beta), \\ \beta \lambda \bar{m}_{2}^{p}(\lambda)-I_{2}^{\beta}\left(\bar{m}_{2}(\lambda)\right), & \lambda>\mathcal{C}_{4}(\beta) .\end{cases}
$$


ДокАЗАтЕЛьСтво. Остается только доказать свойства кривой $\mathcal{C}_{4}(\beta)$.

Если $\beta_{2}<\beta<\beta_{0}$ и $\lambda=\mathcal{C}_{2}(\beta)$, то $\tilde{m}_{2}(\lambda)=m(\beta)$. Если $\lambda$ возрастает, то $\widetilde{m}_{2}(\lambda)$ уменьшается и $\bar{m}_{2}(\lambda)$ увеличивается; таким образом, если $\lambda>\mathcal{C}_{2}(\beta)$, то $\widetilde{m}_{2}(\lambda)<m(\beta)<\bar{m}_{2}(\lambda)$. Но функция $g_{2}(\beta, \lambda ; m)$ является возрастающей для $m \in$ $\left(\widetilde{m}_{2}(\lambda), \bar{m}_{2}(\lambda)\right)$; следовательно, неравенство (54) справедливо. Отсюда следует, что $\mathcal{C}_{4}(\beta) \leqslant \mathcal{C}_{2}(\beta)$

Мы уже видели, что при $\beta=\beta_{2}$ функция $\mathcal{C}_{4}(\beta)$ совпадает с $\mathcal{C}_{2}\left(\beta_{2}\right)=\lambda_{2}$. При $\beta \nearrow \beta_{0}, m(\beta) \searrow 0$, правая часть неравенства $(54)$ стремится к $-I_{2}^{\beta}(0)$. Следовательно, равенство в (54) соответствует (58) (с помощью дифференцирования можно показать, что левая часть (54) является строго возрастающей по $\lambda$ ). Лемма доказана.

Наконец, рассмотрим функцию $f(\beta, \lambda)$. Ясно, что

$$
-\beta f(\beta, \lambda)-\ln 2=\max \left\{F_{1}(\beta, \lambda), F_{2}(\beta, \lambda)\right\} .
$$

Полная фазовая диаграмма изображена на рис. 2 и описывается следующим образом.

Теорема 2. Пусть кривые $\mathcal{C}_{1}(\beta)$ и $\mathcal{C}_{2}(\beta)$ определены уравнениями (38) и (39) соответственно. Пусть $m_{p}$ - единственное положительное решение уравнения $G_{1}(m)=0$, имы положим $\beta_{1}=\bar{\beta}\left(m_{p}\right)$. Если $0<\beta \leqslant \beta_{1}$, то

$$
-\beta f(\beta, \lambda)-\ln 2= \begin{cases}-I_{1}^{\beta}(0)=\beta^{2} /(2 \gamma), & \lambda \leqslant \mathcal{C}_{1}(\beta), \\ \beta \lambda \bar{m}_{1}(\beta, \lambda)-I_{1}^{\beta}\left(\bar{m}_{1}(\beta, \lambda)\right), & \mathcal{C}_{1}(\beta) \leqslant \lambda \leqslant \mathcal{C}_{2}(\beta), \\ \beta \lambda \bar{m}_{2}(\lambda)-I_{2}^{\beta}\left(\bar{m}_{2}(\lambda)\right), & \lambda>\mathcal{C}_{2}(\beta) .\end{cases}
$$

Скачки магнетизачии происходят от 0 до $m_{p}$ поперек кривой $\mathcal{C}_{1}(\beta)$, но магнетизачия постоянна поперек кривой $\mathcal{C}_{2}(\beta)$.

Если $\beta \geqslant \beta_{0}$, mo

$$
-\beta f(\beta, \lambda)-\ln 2= \begin{cases}-I_{2}^{\beta}(0)=\beta \beta_{0} / \gamma-\ln 2, & \lambda \leqslant \lambda_{\mathrm{c}} \\ \beta \lambda \bar{m}^{p}(\lambda)-I_{2}^{\beta}(\bar{m}(\lambda)), \quad \lambda \geqslant \lambda_{\mathrm{c}} .\end{cases}
$$

Скачки магнетизачии происходят от 0 до $m_{\mathrm{c}}$ при $\lambda=\lambda_{\mathrm{c}}$.

При $\beta_{1} \leqslant \beta \leqslant \beta_{0}$ имеется кривая $\mathcal{C}(\beta)$, задаваемая уравнением

$$
\lambda=\mathcal{C}(\beta) \quad \Leftrightarrow \quad-I_{1}^{\beta}(0)=\beta \lambda \bar{m}_{2}^{p}(\lambda)-I_{2}^{\beta}\left(\bar{m}_{2}(\lambda)\right),
$$

$\operatorname{ma\kappa }$ umo $\mathcal{C}\left(\beta_{1}\right)=\mathcal{C}_{2}\left(\beta_{1}\right), \mathcal{C}\left(\beta_{0}\right)=\lambda_{\mathrm{c}} u$

$$
-\beta f(\beta, \lambda)-\ln 2= \begin{cases}-I_{1}^{\beta}(0)=\beta^{2} /(2 \gamma), & \lambda \leqslant \mathcal{C}(\beta), \\ \beta \lambda \bar{m}^{p}(\lambda)-I_{2}^{\beta}(\bar{m}(\lambda)), & \lambda \geqslant \mathcal{C}(\beta) .\end{cases}
$$

ДоказАтельство. Сначала рассмотрим случай $\beta<\beta_{1}$. Для $\lambda \leqslant \mathcal{C}_{2}(\beta)$ имеем

$$
F_{2}(\beta, \lambda)=\beta \lambda m^{p}(\beta)-I_{2}^{\beta}(m(\beta))=\beta \lambda m^{p}(\beta)-I_{1}^{\beta}(m(\beta)) \leqslant F_{1}(\beta, \lambda),
$$

т. е. максимум дается с помощью леммы 5. 
Если $\lambda>\mathcal{C}_{2}(\beta)$, то

$$
F_{1}(\beta, \lambda)=\beta \lambda m(\beta)-I_{1}^{\beta}(m(\beta))=\beta \lambda m(\beta)-I_{2}^{\beta}(m(\beta)) \leqslant F_{2}(\beta, \lambda),
$$

и максимум дается с помощью $F_{2}$, как и в лемме 9 .

При $\beta \geqslant \beta_{0}$ мы имеем $-\beta f(\beta, \lambda)-\ln 2=F_{2}(\beta, \lambda)$. Таким образом, соотношение (71) следует из леммы 9 .

Наконец, рассмотрим случай $\beta_{1}<\beta<\beta_{0}$. Тогда если $\lambda \leqslant \mathcal{C}_{3}(\beta)$, то

$$
-I_{1}^{\beta}(0)>\beta \lambda m^{p}(\beta)-I_{1}^{\beta}(m(\beta))
$$

согласно лемме 5 ; и согласно лемме 9 мы имеем

$$
-\beta f(\beta, \lambda)-\ln 2=\max \left\{-I_{1}^{\beta}(0), \beta \lambda \bar{m}_{2}^{p}(\lambda)-I_{2}^{\beta}\left(\bar{m}_{2}(\lambda)\right)\right\},
$$

т. е. переход дается с помощью $\lambda=\mathcal{C}(\beta)$. В частности, $\mathcal{C}(\beta) \geqslant \mathcal{C}_{4}(\beta)$. Если $\lambda>\mathcal{C}_{3}(\beta)$, то $\lambda>\mathcal{C}_{2}(\beta)$; и так как $\mathcal{C}_{4}(\beta)<\mathcal{C}_{2}(\beta)$, то равенство $(74)$ все еще справедливо.

ПрЕДЛОЖЕНИЕ 2. Кривая $\lambda=\mathcal{C}(\beta)$ является убъвающей и удовлетворяет условиям $\mathcal{C}\left(\beta_{1}\right)=\lambda_{1}$ и $\mathcal{C}\left(\beta_{0}\right)=\lambda_{\mathrm{c}}$. Более того, $\mathcal{C}^{\prime}\left(\beta_{1}\right)=\mathcal{C}_{1}^{\prime}\left(\beta_{0}\right)$ u $\mathcal{C}^{\prime}\left(\beta_{0}\right)=0$.

ДоказАтельство. Мы имеем $\lambda=\mathcal{C}(\beta) \Leftrightarrow D(\beta, \lambda)=0$, где

$$
D(\beta, \lambda)=-I_{1}^{\beta}(0)-\left[\beta \lambda \bar{m}^{p}(\lambda)-I_{2}^{\beta}(\bar{m}(\lambda))\right] .
$$

Отсюда

$$
\mathcal{C}^{\prime}(\beta)=-\left(\frac{\partial D}{\partial \beta}\right)_{\lambda} /\left(\frac{\partial D}{\partial \lambda}\right)_{\beta} .
$$

Используя соотношение $\partial I_{2}^{\beta} / \partial \beta=-\bar{\beta} / \gamma$, мы находим

$$
\frac{\partial D}{\partial \beta}=\frac{\beta-\bar{\beta}(\bar{m}(\lambda))}{\gamma}-\lambda \bar{m}^{p}(\lambda), \quad \frac{\partial D}{\partial \lambda}=-\beta \bar{m}^{p}(\lambda)
$$

откуда получаем

$$
\mathcal{C}^{\prime}(\beta)=\frac{1}{\beta}\left[\frac{\beta-\bar{\beta}(\bar{m}(\lambda))}{\gamma \bar{m}^{p}(\lambda)}-\lambda\right] \leqslant \frac{1}{\beta}\left[\frac{\beta_{0}-\bar{\beta}(\bar{m}(\lambda))}{\gamma \bar{m}^{p}(\lambda)}-\lambda\right]=\frac{1}{\beta}\left[\lambda_{\mathrm{c}}-\lambda\right]<0 .
$$

При $\beta=\beta_{1}$ мы имеем $\mathcal{C}_{1}\left(\beta_{1}\right)=\mathcal{C}_{2}\left(\beta_{1}\right)=\lambda_{1}$. Также ясно, что при $\beta=\beta_{1}$ соотношение $(72)$ справедливо, так как $m\left(\beta_{1}\right)=\bar{m}_{1}\left(\lambda_{1}\right)=\bar{m}_{2}\left(\lambda_{1}\right)$. Тогда $\mathcal{C}\left(\beta_{1}\right)=\lambda_{1}$. При $\beta=\beta_{1}$ справедливо $\bar{m}_{2}(\lambda)=m(\beta)$, следовательно, $\beta=\bar{\beta}$. Отсюда имеем

$$
\left.\mathcal{C}^{\prime}(\beta)\right|_{\beta=\beta_{1}}=-\frac{\lambda}{\beta_{1}} .
$$

Также при $\beta=\beta_{1}$ получаем

$$
\left(\frac{\partial D}{\partial \beta}\right)_{\lambda}=-\lambda \bar{m}_{1}^{p}(\lambda), \quad\left(\frac{\partial D}{\partial \lambda}\right)_{\beta=\beta_{1}}=-\beta_{1} \bar{m}_{1}^{p}(\lambda),
$$

так что $\left.\mathcal{C}_{1}^{\prime}(\beta)\right|_{\beta=\beta_{1}}=-\lambda / \beta_{1}$.

При $\beta=\beta_{0}$ мы находим

$$
\left.\mathcal{C}^{\prime}(\beta)\right|_{\beta=\beta_{0}}=\frac{1}{\beta_{0}}\left[\frac{\beta_{0}-\bar{\beta}(\bar{m}(\lambda))}{\gamma \bar{m}^{p}(\lambda)}-\lambda\right]=\frac{1}{\beta_{0}}\left[\lambda_{\mathrm{c}}-\lambda\right] .
$$

Но при $\beta=\beta_{0}$ мы имеем $\lambda=\lambda_{\text {c }}$, следовательно, $\mathcal{C}^{\prime}\left(\beta_{0}\right)=0$. 


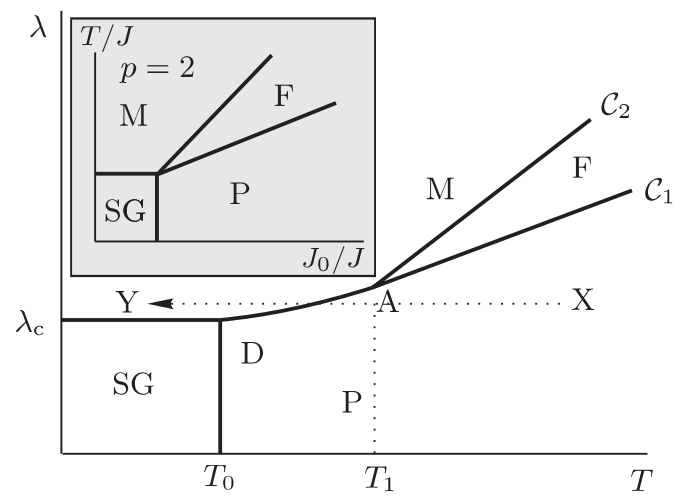

a

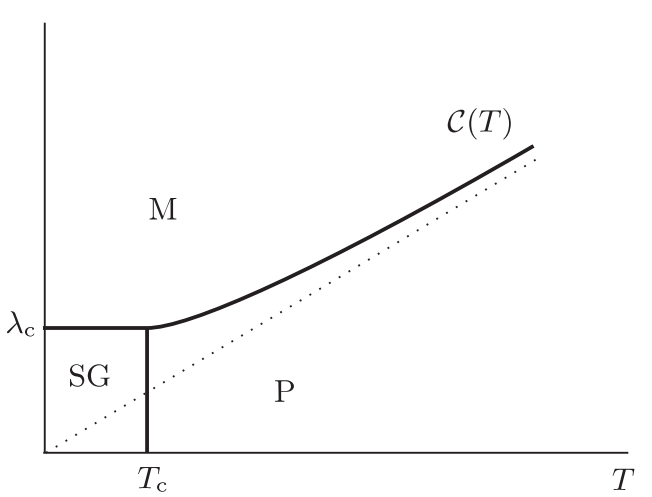

6

Рис. 6. Фазовая диаграмма для $2<p<\infty, T_{0}=1 / \sqrt{2 \gamma \ln 2}$; на вставке приведена фазовая диаграмма для $p=2$ (а). Если температура понижается вдоль стрелки XY из парамагнитной области, то нельзя попасть в ферромагнитную область при больших $p$, так как точки А и D расходятся. В ходе этого анализа обнаружился новый переход поперек кривой $\mathrm{AD}$ при $p>2$, который не существовал при $p=2$. Фазовая диаграмма при $p=\infty$ : упорядоченный ферромагнитный режим далек от достижения (б). Обозначения см. на рис. 2.

\section{5. ФАЗОВАЯ ДИАГРАММА В ПРЕДЕЛЕ $p \rightarrow \infty$}

На рис. 6 изображена фазовая диаграмма, полученная выше, на горизонтальной оси которой отложена температура $T$ вместо обратной температуры $\beta$.

Согласно этой фазовой диаграмме, если понижать температуру по направлению от парамагнитной области при $\lambda$, близком к $\lambda_{c}$, то встречается линия перехода между парамагнитной фазой и смешанной фазой, а не линия между парамагнитной фазой и ферромагнитной фазой. При $p \rightarrow \infty$ это является единственной возмож-

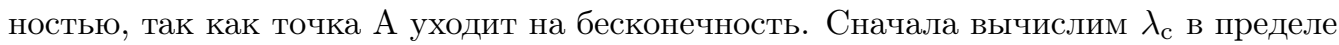
$p \rightarrow \infty$ с помощью соотношения (56). Мы имеем $m_{\mathrm{c}}^{p} \sim\left(1-1 / p^{2}\right)^{p} \rightarrow 1$ при $p \rightarrow \infty$. Следовательно, с учетом (59) мы находим

$$
\lambda_{\mathrm{c}}=\frac{\beta_{0}-\bar{\beta}\left(m_{\mathrm{c}}\right)}{\gamma m_{\mathrm{c}}^{p}} \rightarrow \frac{\beta_{0}}{\gamma}=\frac{\sqrt{2 \ln 2}}{\gamma}
$$

при $p \rightarrow \infty$. Также нетрудно видеть (учитывая $(35),(37)$ и $(38)$ ), что $\mathcal{C}_{1}(\beta) \rightarrow$ $\ln 2 / \beta=T \ln 2$, так как $m_{p} \nearrow 1$ в лемме 2 при $p \rightarrow \infty$. В результате простых вычислений мы получаем

$$
m(\beta)=\operatorname{th}[T(\beta)] \sim 1-\frac{\beta^{2}}{2 \gamma^{2}},
$$

где $m(\beta)$ определено в $(34)$. Теперь $\left(T_{1}, \lambda\left(T_{1}\right)\right)$ является точкой пересечения двух кривых $\mathcal{C}_{1}(T)$ и $\mathcal{C}_{2}(T)$. Это дается решением уравнения $(40)$. Следуя приведенному выше аргументу, получаем $\beta_{1} / \gamma=2^{-(p-1)}$, откуда следует, что при $p \rightarrow \infty$ мы имеем $\beta_{1} \rightarrow 0$, и, следовательно, $T_{1} \rightarrow \infty$. Так как в пределе $p \rightarrow \infty$ справедливо 
$\mathcal{C}_{1}(T)=T \ln 2$, то это означает, что точка пересечения двух кривых $\mathcal{C}_{1}$ и $\mathcal{C}_{2}$ (точка $\mathrm{A}$ ) уходит на бесконечность. Это ясно из фазовой диаграммы для случая $p=\infty$, который показан на рис. 6б. Здесь $\mathcal{C}(T)=T \ln 2+1 /\left(2 T / \gamma^{2}\right)$. Заметим, что в смешанной фазе все еще имеется магнетизация, близкая к единице (даваемая с помощью $m_{\mathrm{c}}$ ), которая сходится к единице не экспоненциально быстро, а приблизительно как $1 / p^{2}$.

\section{6. ОБСУЖДЕНИЕ}

В настоящей статье мы получили фазовую диаграмму модели, определяемой гамильтонианом (7). Фазовые диаграммы для случаев $p=2,2<p<\infty$ и для предельного случая $p \rightarrow \infty$ оказываются разными, так как новая кривая переходов (кривая $\mathcal{C}$ на рис. 2 и кривая DA на рис. 6) появляется при $p>2$, что приводит к уменьшению упорядоченной ферромагнитной фазы при $p \rightarrow \infty$.

Изложим кратко два практических применения этой модели.

Рассмотрим магнитную систему, состоящую из $N$ взаимодействующих спинов Изинга, предполагая, что спиновые значения равны \pm 1 (спиновое стекло). Пусть энергетически благоприятным (ферромагнитным) начальным состоянием системы является состояние $(1,1, \ldots, 1)$. В результате процесса отжига система переходит в более высокое энергетическое состояние, например в состояние $(1,0,1,0,0, \ldots, 1)$. Задача, которую надо решить, заключается в том, как восстановить начальное состояние с помощью подобного процесса отжига. Это фундаментальная проблема в теории информации, впервые сформулированная Шенноном [28], [29]. Возможность переноса оптимального декодирования начального состояния системы на проблему отжига из фазы спинового стекла в ферромагнитную фазу на фазовой диаграмме для $p \geqslant 2$ в модели, которую мы исследовали в настоящей статье, сначала обсуждалась Сурласом [30], [31] и затем анализировалась Дорласом и Ведагедерой [32]-[34]. Оказалось, что это невозможно, так как ферромагнитная фаза полностью исчезает на фазовой диаграмме в пределе $p \rightarrow \infty$. Однако до тех пор, пока мы имеем взаимодействия конечной $p$-группы спинов, можно найти упорядоченные основные состояния системы, если $T \gtrsim T_{1}$ и $\lambda \gtrsim \lambda_{\text {с }}$ (рис. 6 ).

Случай $p<\infty$ оказывается существенным в некоторых исследованиях в области проблемы иммунологии (см. [13]). Действительно, мы могли бы использовать гамильтониан этой модели, чтобы моделировать взаимодействие между рецептором Т-клетки (основная иммунологическая молекула в иммунной клетке называется Т-клеткой) и молекулой пептид-связывающего главного комплекса тканевой совместимости (ПГКТС), которая находится на поверхности антиген-представляющей клетки, и чтобы объяснить с точки зрения статистической механики проблему активации Т-клетки. Действительно, в этой задаче каждая пара, состоящая из рецептора Т-клетки и ПГКТС, берется для образования модели с идеализированными бинарными взаимодействиями $p$-группы, с помощью которых проблема может трансформироваться в задачу, связанную с направленным полимером, которую мы решили в настоящей статье. В реальных биологических случаях $2 \leqslant p \leqslant 10$ (см., например, [35], [36]). Так как при этом изучается поведение модели при $p<\infty$, строгое обоснование фазовой диаграммы в этом случае обеспечивает жизненно важную информацию, связанную с приведенной выше задачей моделирования.

Благодарность. Настоящая работе поддержана National Research Council of Sri Lanka (грант NRC/05/64). 


\section{Список литературы}

[1] É. Brunet, B. Derrida, D. Simon, Phys. Rev. E, 78:6 (2008), 061102, 9 pp., arXiv: 0806.1603.

[2] J.-P. Bouchaud, M. Potters, Theory of Financial Risk and Derivative Pricing, Cambridge Univ. Press, Cambridge, 2003.

[3] J. T. Chayes, L. Chayes, Comm. Math. Phys., 105:2 (1986), 221-238.

[4] E. W. Montroll, G. H. Weiss, J. Math. Phys., 6:2 (1965), 167-181.

[5] W. Paul, J. Baschnagel, Stochastic Processes. From Physics to Finance, Springer, Berlin, 1999.

[6] D. B. Saakian, J. F. Fontanari, Phys. Rev. E, 80 (2009), 041903, 12 pp., arXiv: 0905.1097.

[7] S. Kauffman, S. Levin, J. Theoret. Biol., 128:1 (1987), 11-45.

[8] T. A. Witten, Jr., L. M. Sander, Phys. Rev. Lett., 47:19 (1981), 1400-1403.

[9] C. Giraud, Comm. Math. Phys., 223:1 (2001), 67-86.

[10] Ya. G. Sină, Comm. Math. Phys., 148:3 (1992), 601-621.

[11] D. Dunn-Walters, C. Thiede, B. Alpen, J. Spencer, Philos. Trans. R. Soc. Lond. Ser. A, 29:356 (2001), 73-82.

[12] J. Sun, D. J. Earl, M. W. Deem, Modern Phys. Lett. B, 20:2-3 (2006), 63-95.

[13] J.R. Wedagedera, Directed polymers to model TCR-pMHC interaction, http://www.math.ruh/ac.lk/ janak/research/2010-2.pdf (2010).

[14] B. Derrida, R. B. Griffths, R. G. Higgs, Europhys. Lett., 18:4 (1992), 361-366.

[15] B. Derrida, P. G. Higgs, J. Phys. A, 27:16 (1994), 5485-5493.

[16] S. Havlin, D. Ben-Avraham, Adv. in Phys., 51:1 (2002), 187-292.

[17] B. Derrida, M. R. Evans, E. R. Speer, Comm. Math. Phys., 156:2 (1993), 221-244.

[18] É. Brunet, B. Derrida, Phys. Rev. E, 61:6 (2000), 6789-6801, arXiv: cond-mat/0005352.

[19] B. Derrida, Phys. Rev. B, 24:5 (1981), 2613-2626.

[20] B. Derrida, J. Phys. Lett., 46:9 (1985), 401-407.

[21] É. Brunet, B. Derrida, D. Simon, Phys. Rev. E, 78:6 (2008), 061102, 9 pp., arXiv: 0806.1603.

[22] B. Derrida, Phys. Rev. Lett., 45:2 (1980), 79-82.

[23] J. R. Wedagedera, T. C. Dorlas, J. Statist. Phys., 103:5-6 (2001), 697-716.

[24] E. Buffet, A. Patrick, J. V. Pulé, J. Phys. A, 26:8 (1993), 1823-1834.

[25] J. R. Wedagedera, Mathematical aspects of some mean field spin glass models, Ph.D. Thesis, Univ. of Wales, Swancea, 1998.

[26] D. Williams, Probability with Martingales, Cambridge Math. Textbooks, Cambridge Univ. Press, Cambridge, 1991.

[27] S. R. S. Varadhan, Large Deviations and Applications, SIAM, Philadelphia, 1984.

[28] C. E. Shannon, Bell System Tech. J., 27 (1948), 379-423; 623-656.

[29] C. E. Shannon, W. Weaver, A Mathematical Theory of Communication, Univ. of Illinois Press, Urbana, 1963.

[30] N. Sourlas, Nature, 339:6227 (1989), 693-695.

[31] N. Sourlas, "Statistical mechanics and error-correcting codes", Statistical Mechanics of Neural Networks, Lecture Notes in Phys., 368, ed. L. Garrido, Springer, Berlin, 1990, $317-330$.

[32] T. C. Dorlas, J. R. Wedagedera, Phys. Rev. Lett., 83:21 (1999), 4441-4444.

[33] T. C. Dorlas, J. R. Wedagedera, Internat. J. Modern Phys., 15:1 (2001), 1-15.

[34] P. Gillin, D. Sherrington, H. Nishimori, J. Phys. A, 34:14 (2001), 2949-2964, arXiv: cond-mat/0103292.

[35] D. J. Irvine, M. A. Purbhoo, M. Krogsgaard, M. M. Davis, Nature, 419 (2002), 845-849.

[36] C. Wülfing, J. D. Rabinowitz, C. Beeson, M. D. Sjaastad, H. M. McConnell, M. M. Davis, J. Exp. Medicine, 185:10 (1997), 1815-1825. 